\title{
La evolución del sector televisivo: un oligopolio frente a Internet
}

\author{
Jessica IZQUIERDo-CASTILlo \\ Universitat Jaume I \\ jizquier@uji.es
}

\begin{abstract}
Resumen:
La televisión en España se enfrenta a un momento de incertidumbre en la transición al espacio convergente. El análisis de la estructura del sector televisivo desde una perspectiva histórica puede contribuir a identificar los factores relevantes que marcan la configuración del sector. Este artículo presenta un análisis de la evolución del sector atendiendo a tres grandes periodos. En primer lugar, la privatización de la televisión en España y el desarrollo de un sector competitivo. En segundo lugar, la multiplicación de la oferta en el contexto digital. Por último, el proceso de renovación propiciado por Internet y las nuevas formas de consumo de contenidos televisivos de la audiencia.
\end{abstract}

Palabras clave: televisión; estructura; evolución; tecnología; política.

\section{The Evolution of the Television Industry: an Oligopoly Facing Internet}

\begin{abstract}
:
Television in Spain is facing a time of uncertainty in the transition to the converged scenario. The structural analysis of this industry from a historical perspective can help identify key factors in the configuration of the sector. This paper presents an analysis of the evolution of the sector according to three main periods. Firstly, it approaches the privatization of the television in Spain and the development of a competitive sector. Secondly, it studies the increase in supply in the digital context. Finally, it focuses on the renewal process facilitated by the Internet and new forms of consumption of television content from the audience.
\end{abstract}

Key Words: television; structure; evolution; technology; politics.

\section{Referencia normalizada:}

Izquierdo-Castillo, J. (2014): La evolución del sector televisivo: un oligopolio frente a internet. Historia y Comunicación Social. Vol. 19. Núm. Especial Enero. Págs. 735-745.

Sumario: 1. Introducción. 2. La configuración de una estructura privada. 3. la digitalización de la TDT. 4. Internet y la televisión. 5. Conclusiones. 6. Referencias. 


\section{Introducción}

El sector televisivo se ha caracterizado por estar bajo una estructura en constante evolución, al igual que el resto del sector comunicativo. En la actualidad, la digitalización y, concretamente la convergencia en Internet, abre la puerta a un abismo todavía por abordar. Esto sitúa a la televisión ante una gran incertidumbre. En este contexto, una retrospectiva sobre las etapas evolutivas del sector, diferenciadas dentro del contexto privatizado, permiten trazar una línea evolutiva donde se identifiquen los principales factores que dinamizan la evolución de este sector en constante transformación.

Este artículo tiene como principal objetivo dibujar una línea evolutiva de la estructura de la televisión. Se toma la perspectiva teórica de la Economía Política de la Comunicación y se aplica sobre el sector, con la intención de determinar la incidencia de los factores tecnológico, político y económico, sobre los procesos de cambio en su desarrollo. La hipótesis de partida sitúa a estos factores como principales protagonistas en la configuración el sector y en su evolución, por lo que determina que una visión histórica sobre su papel en las etapas anteriores permitirá delimitar las líneas de desarrollo en el entorno digital. Cada etapa se delimita en torno a los efectos que cada uno de estos factores han causado en su estructuración. Así, desde la dimensión tecnológica se traza un vínculo entre las sucesivas actualizaciones relacionadas con los métodos de distribución (cable, satélite, terrestre), a la digitalización y, especialmente, a la convergencia. Por otra parte, desde la perspectiva económica se analizan los aspectos relacionados con la financiación del sector, así como los resultados de audiencia y su contribución a la configuración del sector. Por último, el factor político determina la estructura televisiva desde las políticas de comunicación y es precisamente este criterio el que se toma para delimitar los periodos de las tres etapas sujetas a análisis. Por tanto, la primera etapa queda delimitada por la aprobación de La ley de las Televisiones Privadas, en 1989. La segunda con la concesión de las primeras licencias para la Televisión Digital terrestre, en 2000. La tercera con el apagón analógico en 2010 y la definitiva digitalización del sector.

Para el análisis del sector desde esta perspectiva histórica es fundamental la recopilación de trabajos de referencia, que han abordado, bien desde la retrospectiva, bien desde la contemporaneidad, la evolución del sector televisivo. Así, autores como Bustamante (2013; 2003; 2002; 1999), Bustamante y Álvarez-Monzoncillo (2011; 1999), Giordano y Zeller (1999), Reig (1998), Baget (1993) o Palacio (1992), entre otros, ofrecen obras de obligada consulta en el campo de la investigación televisiva. Las aportaciones de estos, y otros, autores constituyen una fuente fundamental en la construcción de esta panorámica evolutiva. A partir de estos trabajos, se toma la perspectiva de la Economía Política de la Comunicación, que aplica sobre el estudio las nociones de mercantilización, especialización y estructuración (Mosco, 2009; 2006) para analizar el sistema comunicativo desde la globalización (Bernardo Paniagua, 2006) y la configuración de una sociedad interconectada a escala mundial (Castells, 2006; 2001). Desde este enfoque el análisis se centra en los factores que condicio- 
nan los procesos clave que acompañan a la configuración de un sector comunicativo como el televisivo.

El objetivo de este recorrido por la evolución del sector reside en conocer de forma retrospectiva los elementos que han marcado el desarrollo de la televisión, para determinar cuáles son los factores clave que permitan enfrentar el reto de la convergencia mediática en la etapa actual.

\section{La configuración de una estructura privada}

La privatización del sector televisivo en España se produjo con la Ley de Televisiones Privadas de 1989, después de casi una década de negociaciones e intentos fallidos. Los antecedentes que propiciaron la definitiva aprobación de la ley acumularon sucesivos acercamientos por parte de los principales líderes políticos de los partidos mayoritarios para introducir el modelo privado (Fernández y Santana, 2000), así como denuncias por parte de grupos mediáticos, que presionaban para obtener presencia en el sector televisivo (Artero, 2008). Con la concesión de tres licencias para la emisión en abierto se culminó un proceso de privatización mediática, que había actuado años antes en el sector de la prensa, donde el Estado primero privatizó el sector en 1984 y después eliminó las ayudas en 1988 (Bustamante, 2013).

El resultado de las concesiones creó una nueva estructura, que no sólo se imponía sobre el número de operadoras, sino que tuvo implicaciones sobre el consumo de los telespectadores y su relación con el medio. Las concesionarias que recibieron las licencias fueron: Antena 3 Televisión, Gestevisión Telecinco y la Sociedad Española de Televisión Canal Plus. El reparto no estuvo exento de polémicas y denuncias (Artero, 2008). El reparto final provocó, la acusación hacia el gobierno de haber propiciado la dualidad, con la derecha (Antena 3) y la izquierda (Telecinco) y el partidismo en beneficio de los intereses del gobierno del Partido Socialista Obrero Español (PSOE) (Canal Plus).

Con esta medida política se rompía la estructura monopolística del sector, formada por un reducido sistema compuesto por dos canales públicos y, en el caso de algunas comunidades autónomas, un tercer canal (Miguel de Bustos y Casado, 2012). Pero la decisión no estuvo únicamente propiciada por los actores políticos, sino que también fue determinante la presión de los anunciantes y de los grandes grupos de comunicación (Artero, 2008). Por tanto, el factor económico también influyó en la modificación de la estructura sectorial, además de intervenir en su posterior desarrollo. Así, la publicidad jugó en este periodo determinó la estructura financiera de cada cadena. Los ingresos publicitarios, que había crecido exponencialmente durante la década de 1980, decayeron hasta registrar un crecimiento nulo de 1990 a 1993. La única cadena que creció en este periodo fue Canal Plus, que contaba con una fuente de ingresos adicional con las cuentas de abonados, lo que evitó que acumulara deuda, como el resto de las operadoras. Los malos resultados económicos provocaron la lucha por 
la audiencia y, en concordancia, por la publicidad. Los precios bajaron de manera vertiginosa y esto conllevó un aumento de la presencia de mensajes publicitarios, lo que provocó, como consecuencia, la saturación en las cadenas de emisión en abierto. A partir de 1995, sin embargo, esta situación financiera cambió y las cadenas privadas aumentaron sus ingresos publicitarios y redujeron sus ratios de endeudamiento. Antena 3 registró un crecimiento del 50\% y los ingresos de Telecinco incrementaron un 140\% (Artero, 2008). Por tanto, las privadas obtuvieron, en el periodo 1995-1999 una mayor estabilidad, no sólo financiera (Giordano y Zeller,1999), sino en su accionariado (Bustamante, 2013; Artero, 2008). Sin embargo, la saturación publicitaria no disminuyó y algunos autores advertían de los efectos negativos que eso suponía para el desarrollo del sector televisivo (Medina, 1995; Pérez-Latre, 2000).

La dimensión económica también motivó las decisiones en el ámbito de la televisión de proximidad. Las televisiones autonómicas se unieron el 5 de abril de 1989 para hacer frente al nuevo mercado privado. Los terceros canales, con emisión en un ámbito acotado, formaron la Federación de Organismos de Radio y Televisión Autonómicos (FORTA), con el objetivo de conseguir sinergias económicas. Aún así, estas cadenas desarrollaron una fuerte dependencia financiera con las administraciones públicas, pues en 1991 el $60 \%$ de sus presupuestos globales provenían de la subvenciones autonómicas. Durante la década de 1990, las autonómicas consolidaron su audiencia y el desarrollo positivo del mercado favoreció la progresión de ingresos económicos. En este ámbito, la estructura también se amplió y se aprobaron los segundos canales en algunas autonomías. Respecto a la televisión por cable, la estructura desorganizada y prolífica de las redes locales (Bustamante, 2013) se regularizó en 1995 mediante la Ley de Telecomunicaciones, que establecía la reordenación del sector y se crearon dos grandes grupos de cable: la Asociación de Operadores de Cable (AOC), con Endesa, Telecom Italia y Unión Fenosa como principales accionistas; y Cableuropa, empresa que comercializaba ONO, con el BSCH. Estos grupos podían incorporar como valor añadido "la oferta de servicios plenamente interactivos y [...] la potenciación de contenidos locales" (Arrese, 2000: 105). Sin embargo, tuvieron dificultades para conseguir abonados, ya que uno de los principales reclamos, la exclusividad de los contenidos, ya estaban en manos de las plataformas digitales, que habían conseguido levantar barreras de entrada a la televisión de pago.

Por último, el papel que jugó el factor tecnológico en esta primera fase de implantación de la televisión privada se limitó a la concesión de la infraestructura de titularidad pública para la emisión de los canales privados, según recogía el Plan Técnico de la Televisión Privada. En este ámbito, un Real Decreto de 19 de mayo de 1989 estableció el Ente Público de la Red Técnica Española de Televisión, conocido como Retevisión. También destaca en el ámbito tecnológico el lanzamiento del primer satélite español, Hispasat, con la participación de Telefónica (25\%), Retevisión (25\%), Caja Postal $(22,5 \%)$ e INI (10\%). El gobierno otorgó un canal satélite a cada cadena nacional, que se unieron en la Comercializadora de Televisión por Satélite (Cotelsat), con un capital social de 1.000 millones de pesetas. Esta iniciativa fracasó en 1995, pero un poco más tarde, en 1997, salieron al mercado dos plataformas que conforma- 
rían la principal oferta en este subsector. Sogecable completó la oferta de Canal Plus con Canal Satélite Digital y Telefónica creó Vía Digital. Durante tres años, ambas plataformas se enfrentaron en una guerra por los contenidos, principalmente deportivos, que provocó un baile de accionistas que intentaba frenar las pérdidas económicas, principalmente las de Vía Digital, que no consiguió imponerse a su competencia, que contaba con los abonados de Canal Plus y una amplia biblioteca de derechos de contenidos (Bustamante, 2002).

De forma paralela, mientras el sector televisivo se ampliaba con mayor oferta de canales y nuevas redes de distribución, los hogares españoles se adaptaron al cambio propuesto por el medio y por la tecnología. El equipamiento de los hogares en esta primera etapa pasó de estar formado por un televisor por hogar a contar con dos o más aparatos $(60,2 \%$ hogares en 1999). Además, el televisor se complementó con el mando a distancia y los magnetoscopios, lo que transformó el hábito de consumo televisivo y otorgó poder al usuario.

En grandes líneas, esta etapa representó la configuración de un mercado televisivo comercial, con una estructura pública liderada por un canal estatal mayoritario y dos canales segmentados (La 2 y el tercer canal) y una estructura privada que, tras una serie de movimientos en las estructuras de propiedad de cada cadena privada (Artero, 2008; Bustamante, 2013), quedó incorporada a los intereses de los grandes grupos informativos. De esta forma, estos grupos habían adquirido una posición central en la propiedad y en la gestión de la televisión en España (Díaz Nosty, 1996). De forma paralela, los nuevos sistemas de transmisión de la señal, a través del satélite, asentaban los cimientos de un sector televisivo de pago que se encontraría con dificultades, principalmente motivadas por la incapacidad de absorción del mercado. En definitiva, la televisión privada, la televisión de proximidad, el desarrollo de las nuevas tecnologías de redifusión y los cambios en los hábitos de consumo; son los protagonistas de una década que formó una estructura televisiva dicotómica entre la organizada sector de la televisión generalista y el caos organizativo del sector local. El resultado es una estructura sobredimensionada para un sector que tenía trazado un plan de expansión para su siguiente etapa.

\section{La digitalización de la TDT}

Esta etapa tiene como punto inicial la concesión de las primeras licencias de explotación televisiva digital. En el año 2000 el gobierno concedió dos nuevas licencias para dos cadenas digitales. Las concesionarias fueron Net TV, participada por Prensa Española y un consorcio de productoras (Globomedia, Telson y Cartel) y Veo TV, de Unedisa y Recoletos. Al mismo tiempo, las televisiones privadas renovaron su licencia por otra década, junto con la obligatoriedad de simultanear la emisión con la señal digital en un plazo de dos años. Comenzaba así la implantación de la Televisión Digital Terrestre (TDT). 
España fue uno de los primeros países europeos en adoptar la TDT. Sin embargo, lejos de convertirse en algo positivo, España demostró "en la práctica las nefastas consecuencias de una política industrial hecha a remiendos, sin visión de conjunto y sin tener en cuenta que todos los elementos que integran el sistema audiovisual están interrelacionados" (Prado, 2003: 180). De hecho, las cadenas digitales no conseguían introducirse en el mercado televisivo, y el propio gobierno tuvo que modificar las condiciones de la concesión para facilitar la actividad de Veo TV y Net TV. Respecto a las cadenas analógicas, en febrero de 2005 se procedió al reparto definitivo de canales, después de algunas modificaciones (Artero, 2008). Además, ese año se otorgó una nueva licencia analógica, concedida para La Sexta, conformada por Televisa $(40 \%)$ y un consorcio de productoras españolas $(60 \%)$ entre las que destaca Globomedia y Mediapro. Además, la decisión iba acompañada de una modificación de la licencia de 1989 de Canal Plus, que se convertía en abierto con el canal Cuatro. Por lo tanto, el mapa televisivo con cobertura estatal pasó de estar formado por cuatro actores (RTVE, Telecinco, Antena 3 y Canal Plus) a ocho (Net TV, Veo TV, Cuatro y La Sexta). El mapa televisivo digital tras el apagón analógico en 2010, quedaría formado por un múltiplex (4 canales) para cada televisión privada y dos múltiplex para TVE. Por otra parte, la concesión de licencias digitales para la televisión local estuvo marcada por las políticas polémicas y conformaron un mapa poco profesional y de marcado interés partidista (Bustamante, 2013).

El ámbito político potenció la multiplicación del sector televisivo durante esta década. Los efectos de este crecimiento incidieron sobre el ámbito económico. Por si el mapa de cadenas no resultaba abrumador, para un mercado español de tamaño reducido, la audiencia también podía elegir entre: las corporaciones autonómicas y sus cuatro canales digitales concedidos; las locales, agrupadas en dos grandes opciones (AOC y Cableuropa); y la televisión por satélite, también con dos plataformas (Canal Satélite Digital y Vía Digital). En lo referente a la televisión autonómica, el desarrollo de la audiencia alcanzó cierta estabilidad entre 1999 y 2004, pero ningún canal lideraba en su mercado. Los niveles de cuota de mercado se vieron afectados por la fragmentación en la segunda mitad de la década y la audiencia decayó hasta un nivel mínimo en 2010. Esto provocó que algunas cadenas, como la de Canarias o Madrid, intentaran aplicar medidas de semiprivatización (Artero, 2008: 231) que no se llevaron a cabo. La paradoja la protagonizaban, sin embargo, algunas comunidades autónomas, que abrían su propia cadena de televisión, por un lado y, por otro, la aparición de los segundos canales para aquellas con mayor tradición televisiva. Mientras tanto, el mercado de la televisión de pago no era capaz de albergar a tantos operadores. La ventaja de Canal Satélite Digital frente a la débil Vía Digital provocó su absorción en 2002. Tres años después, las dos plataformas de cable se fusionaron para hacer frente a la competencia directa de Telefónica, que se acababa de introducir en el mercado televisivo, después de afianzar su posición en la telefonía, y por la fuerte inversión que suponía la construcción de la infraestructura de fibra óptica. Por su parte, la televisión local experimentó un giro durante el periodo entre $2000 \mathrm{y}$ 2005. Las empresas informativas se interesaron por la televisión y crearon un modelo similar al radiofónico. Los grupos Prisa, Vocento y COPE, crearon redes de emisoras 
locales que podían llegar a obtener cobertura estatal. Algunos autores preveían la importancia que podía alcanzar esta expansión (Medina, 2005), pero el mercado de la televisión local no se afianzó (Marzal y Casero, 2008)

Desde el ámbito tecnológico, esta etapa estuvo marcada por la transición de la televisión terrestre del escenario analógico al digital. Los sistemas de transmisión de la señal implicaron una renovación en todos los emisores. Sin embargo, la renovación más revolucionaria se impuso sobre los receptores. Los telespectadores se vieron obligados a adaptar sus sistema de recepción de la señal a los requerimientos digitales. De esta forma, se modificó el parque de antenas y se abrió el parque de descodificadores. La renovación tecnológica comportaba el planteamiento de ciertos retos, como la interactividad asociada a la televisión.

En esta etapa la televisión queda configurada por una estructura cuya dimensión excede la capacidad de absorción del mercado y no existe un organismo independiente que vigile y regule la viabilidad y el desarrollo del sector, tanto a nivel estructural como su funcionamiento. El comienzo de la siguiente etapa cuestiona la multiplicación de la oferta, con resultados de fragmentación, así como denota una incongruencia en el comportamiento del sector respecto a los hábitos del telespectador y la tecnología de acceso a los contenidos.

\section{Internet y la televisión}

La tercera etapa comienza con el punto de inflexión que supuso el "apagón analógico" para la estructura televisiva, el 3 de abril de 2010. El crecimiento de la etapa anterior provocó la aparición de nuevos operadores, de naturaleza exclusivamente digital (Net TV, Veot TV), así como la multiplicación de canales a través de los multiplex. En esta etapa el sector televisivo privado asiste al desarrollo de tres fenómenos: la desregulación, manifiesta en la rebaja de la obligación sobre las operadoras televisiva para la inversión en cine o la retirada de la publicidad en TVE; un proceso de concentración, con la fusión de Telecinco y Cuatro (2010) y de Antena 3 y La Sexta (2012); y una tendencia hacia la internacionalización, con la presencia de accionariado extranjero en las cadenas privadas (Bustamante, 2013: 265, 273-274).

Desde el ámbito político, la llegada del Partido Popular al poder se tradujo de forma casi inmediata en importantes modificaciones sobre la televisión pública. Por ejemplo, en la elección del Presidente del Consejo, para el que bastaría la mayoría simple del Parlamento. Este proceso de regubernamentalización (Bustamante, 2013: 307) también está patente en la aprobación de la privatización o externalización de las televisiones autonómicas. Por su parte, la televisión local acusó la errática dispensación de las licencias de la etapa anterior, gobernada por el Partido Socialista Obrero Español y el subsector se quedó sin un modelo viable de desarrollo. A pesar de ello, este "fracaso ostentoso no originó ninguna reacción del Gobierno ni intento alguno 
de reutilizar esas licencias para fomentar una auténtica televisión de proximidad" (Bustamante, 2013:279).

Desde el ámbito económico, la primera consecuencia para el mercado televisivo fue la fragmentación de audiencias, que provocó la pérdida de veinte puntos porcentuales en el share de las tres cadenas principales (TVE, Telecinco y Antena 3). Sin embargo, el acumulado de audiencias por grupo televisivo permanece con un reparto de cuotas similares entre los principales operadores. De hecho, los movimientos de concentración provocan que la estructura oligopólica de la segunda etapa se transforme en un "oligopolio imperfecto, o incluso en un duopolio claro en el mercado televisivo" (García Santamaría y Fernández-Beaumont, 2012: 58) con Mediaset y Atresmedia. Además, la inversión publicitaria desciende cada año y eso plantea interrogantes sobre la producción de los contenidos. Ante esta coyuntura, algunos actores están aplicando estrategias de autosuficiencia, siguiendo el modelo de plataformas de Internet nativas, como Netflix (Izquierdo, 2012). Así, Telefónica ha anunciado el lanzamiento de Telefónica Studios, que se encargará de producir contenidos para su televisión por cable. En este campo, los operadores de pago están realizando avances, como Canal Plus y su plataforma Yomvi. Los operadores privados que emiten en abierto también experimentan con fórmulas, como Mediaset y su plataforma Mitele, o la plataforma Nubeox de Atresmedia. Sin embargo, ninguna de estas iniciativas está asentada en un modelo de negocio sostenible, ya que su introducción en Internet está motivada por una necesidad y no una estrategia de expansión de negocio.

Sin embargo, el principal factor de cambio en esta etapa es el tecnológico. El proceso de desarrollo e implantación de la TDT en la etapa anterior se realizó sin seguir la directiva europea que determinaba reservar el espacio radioeléctrico de la frecuencia que ahora utiliza la TDT en España para las comunicaciones móviles de banda ancha. Esto implica una renovación del parque de receptores y una adaptación de los emisores. A pesar de los inconvenientes que este proceso pueda causar en el mercado televisivo, esto no se presenta como un factor fundamental en la configuración estructural del sector. En este sentido, a diferencia de las etapas anteriores, donde las transformaciones técnicas son intrínsecas a la televisión, en la etapa actual es una tecnología externa la que provoca la presión sobre la estructura televisiva. Se trata de Internet y su papel convergente sobre el conjunto del sistema comunicativo. Así, los contenidos televisivos ya no están sujetos a la transmisión de la señal mediante redes de difusión, sino que se han trasladado a un espacio donde los operadores pierden el control absoluto sobre ellos. Esta transformación obliga a las empresas mediáticas a realizar una transición hacia el espacio convergente, impulsados por la autonomía creciente del usuario a consumir sus contenidos. En este sentido, los operadores televisivos han experimentado con diversas fórmulas para introducirse en Internet y seguir controlando el acceso (Izquierdo, 2012). Sin embargo, han surgido nuevas plataformas que intervienen en la mediación de los contenidos y que no están vinculadas a las operadoras televisivas. 


\section{Conclusiones}

El planteamiento inicial para la selección de los puntos de inflexión entre etapas condiciona el enfoque de este recorrido panorámico por los factores que marcan la evolución histórica de la estructura del sector televisivo en España. No obstante, parece que el factor político es, junto con el económico, el principal motor de transformación de los componentes del sector. Las decisiones políticas en materia televisiva se encuentran ligadas a tres intereses: el servicio público, la relación con el mercado y el control político de los medios. La cuestión del servicio público del sistema televisivo se encuentra en uno de los momentos más delicados de la historia democrática de España. Durante el periodo analizado, las actuaciones políticas han demostrado niveles de preocupación dispares, pero, en cualquier caso, no se ha trabajado con esfuerzo en la aplicación de medidas que permitan la regulación y el control independiente del sistema comunicativo, al margen de los intereses políticos. Sin embargo, la búsqueda por el control comunicativo y las presiones de los grupos de comunicación han constituido uno de los principales aspectos influyentes en las sucesivas medidas políticas.

Por su parte, el factor tecnológico ha protagonizado una función más discreta en la configuración de la estructura televisiva. Así, durante el periodo analizado, su función ha recaído en la apertura de nuevas vías de difusión de contenidos, ampliando mercado televisivos en el cable, el satélite o la TDT. Sin embargo, en la tercera etapa su influencia sobre el sistema comunicativo en su conjunto adquiere una relevancia fundamental. La aparición de Internet y su capacidad de convergencia de todos los medios, obliga a sectores como la televisión a integrarse en un nuevo universo, donde los operadores tradicionales tienen dificultades para mantener su hegemonía. Por tanto, es el factor tecnológico el que provoca que la televisión se enfrente, en la etapa actual, a un escenario donde la los usuarios poseen la capacidad de ser ellos quienes deciden su papel como telespectadores. Las normas que rigen la relación entre la televisión y el espectador ya no son válidas en el contexto del vídeo bajo demanda y los dispositivos móviles. Además, nuevos actores aparecen en el contexto convergente y plantean modelos de negocio que obligan a los operadores televisivos a proponer sus propios proyectos. Todo esto abre interrogantes, no sólo por la evolución de la televisión, sino por la viabilidad de los contenidos que produce y difunde. Hasta ahora, las medidas políticas han regulado sobre el sector televisivo, con innegables efectos en la configuración de su estructura. Sin embargo, Internet no permite la actuación delimitadora de las políticas públicas, tradicionalmente ligadas a la concesión de licencias de emisión, ya que no pide exclusión en la difusión de señal. No obstante, los contenidos están limitados a productoras que, por otra parte, encuentran competencia en las propias plataformas online de distribución de contenidos.

En definitiva, los parámetros tradicionales, que se han aplicado al sector televisivo, parecen no ser de la misma utilidad en el escenario online. En este marco, los operadores de telecomunicaciones han adquirido mayor protagonismo, por el control de las redes de distribución. Sin embargo, la pauta que modela la oferta está, más que 
nunca, bajo los comportamientos de la audiencia. Los actores económicos y políticos tradicionales, recogidos en la publicidad, los grupos de comunicación y los gobiernos nacionales y autonómicos, pierden fuerza ante la incontestable hegemonía tecnológica y los usos de la audiencia. No obstante, Internet es todavía un escenario donde la oferta de contenidos todavía no ha encontrado una estructura formal o, lo que es más necesario, un modelo de negocio rentable para el mercado español.

\section{Bibliografía}

ARRESE, A. (2000). Servicios de difusión televisiva. En: DURÁNDEZ, Ángel \& SÁNCHEZ-TABERNERO, A. (dir.): El futuro de la televisión en España. Análisis prospectivo (2000-2005). Madrid: Arthur Andersen y Universidad de Navarra.

ARTERO, J.P. (2008). El mercado de la televisión en España: oligopolio. Barcelona: Deusto.

BAGET, J.M. (1993). Historia de la televisión en España (1956-1975). Barcelona: Feed-Back, D.L.

BERNARDO PANIAGUA, J.M. (2006). El Sistema de la comunicación mediática: de la comunicación interpersonal a la comunicación global. Valencia: Tirant lo Blanch. p. 304-310

BUSTAMANTE, E. (2013). Historia de la radio y la televisión en España. Una asignatura pendiente de la democracia. Barcelona: Gedisa.

BUSTAMANTE, E. (coord.) (2003). Hacia un nuevo sistema mundial de comunicación: industrias culturales en la era digital. Barcelona: Gedisa.

BUSTAMANTE, E. ( coord.) (2002). Comunicación y cultura en la era digital: industrias, mercados y diversidad en España. Barcelona: Gedisa.

BUSTAMANTE, E; ÁLVAREZ MONZONCILLO, J.M. (eds.) (2011). La televisión etiquetada: nuevas audiencias, nuevos negocios. Madrid: Ariel.

BUSTAMANTE, E; ÁLVAREZ MONZONCILlO, J.M. (1999) (eds.). Presente y futuro de la televisión digital. Madrid: Edipo.

BUSTAMANTE, E. (1999). La televisión económica. Financiación, estrategia y mercados. Barcelona: Gedisa.

CASTELLS, M. (2006). Información, redes y sociedad red: una propuesta teórica. En: Castells, M. La sociedad red: una visión global. Madrid: Alianza Editorial. p. $27-75$

DE BUSTOS, J.C.; CASADO DEL RÍO, A. (coords.) (2012). Televisiones autonómicas: evolución y crisis del modelo público de proximidad. Barcelona: Gedisa

DÍAZ NOSTY, B. (2000)(ed.). Informe anual de la comunicación 1999-2000. Barcelona: Zeta-Endesa.

FERNÁNDEZ, I.; SANTAN, F. (2000). Estado y medios de comunicación en la España democrática. Madrid: Alianza.

GARCÍA SANTAMARÍA, J.V. y FERNÁNDEZ-BEAUMONT, J. (2012). Concentración y pluralismo ene 1 nuevo escenario audiovisual español. Análisis de la absorción de la cadena Cuatro por Telecinco. En: Anàlisi, 47. p.57-74 
GIORDANO, E. y ZÉLLER, C. (1999). Politicas de televisión. La configuración del mercado audiovisual. Barcelona: Icaria.

IZQUIEDO, J. (2012). Distribución de contenidos audiovisuales: análisis de 3 modelos de negocio. En: El profesional de la información, v.21, nº 4, julio-agosto.

MARZAL, J. y CASERO, A. (2008). La investigación sobre la televisión local en España: nuevas agendas ante el reto de la digitalización. En: Zer: Revista de estudios de comunicación, $\mathrm{n}^{\circ} 25$. p.83-106.

MEDINA LAVERÓN, M. (2005). Estructura y gestión de empresas audiovisuales. Pamplona: EUNSA

MEDINA LAVERÓN, M. (1995). El mercado de la televisión terrestre: cinco años en perspectiva. En: SÁNCHEZ-TABERNERO, A. (coord.). La industria de la comunicación, Informe Situación. Madrid: BBV.

MOSCO, V. (2006). La Economía Política de la Comunicación: una actualización diez años después. En: Cuadernos de Información y Comunicación, vol. II. p. $57-79$

PALACIO, M. (2001). Historia de la televisión en España. Barcelona: Gedisa.

PALACIO, M. (1992). Una historia de la televisión en España: arqueología y modernidad. Madrid: ELR.

REIG, R. (1998). Medios de comunicación y poder en España. Prensa, radio, televisión y mundo editorial. Barcelona: Paidós.

PÉREZ-LATRE, F.J. (2000). Publicidad. En: DURÁNDEZ, Ángel \& SÁNCHEZ-TABERNERO, A. (dir.). El futuro de la televisión en España. Análisis prospectivo (2000-2005). Arthur Andersen y Universidad de Navarra. Madrid.

PRADO, E. (2003). Virtudes, funciones y futuro de la TDT en la Sociedad de la Información. En: Telos, ${ }^{\circ}$ 57. Madrid: Fundación Telefónica.

TABERNERO, A. (dir.). El futuro de la televisión en España. Análisis prospectivo (2000-2005). Madrid: Arthur Andersen y Universidad de Navarra.

\section{La autora}

Es profesora en la Universitat Jaume I (UJI) de Castellón. Es doctora en Comunicación audiovisual y licenciada en Publicidad y RRPP por la UJI y en Comunicación audiovisual por la Universitat de València. Es miembro de los grupos de investigación Investigación en tecnologías aplicadas a la comunicación (Itaca) y Periodismo, comunicación y poder, en el Departamento de Ciencias de la Comunicación de la UJI. Sus líneas de investigación se centran en la estructura del sistema comunicativo en el escenario digital, el ámbito de la programación televisiva y los estudios sobre el desarrollo de modelos de negocio, especialmente en Internet, para la prensa y los contenidos audiovisuales. 